\title{
A QCD SUM RULE STUDY FOR A CHARGED BOTTOM-STRANGE SCALAR MESON
}

\author{
C.M. Zanetti ${ }^{1}$, M. Nielsen ${ }^{2}$ and K.P. Khemchandani ${ }^{1}$ \\ ${ }^{1}$ Faculdade de Tecnologia, Universidade do Estado do Rio de Janeiro, \\ Rod. Presidente Dutra Km 298, Pólo Industrial, 27537-000, Resende, RJ, Brasil \\ ${ }^{2}$ Instituto de Física, Universidade de São Paulo, C.P. 66318, 05389-970 São Paulo, SP, Brazil
}

\begin{abstract}
Using the QCD sum rule approach we investigate the possible four-quark structure for the new observed $B_{s}^{0} \pi^{ \pm}$narrow structure (D0). We use a diquak-antidiquark scalar current and work to the order of $m_{s}$ in full QCD, without relying on $1 / m_{Q}$ expansion. Our study indicates that although it is possible to obtain a stable mass in agreement with the state found by the D0 collaboration, a more constraint analysis (simultaneous requirement of the OPE convergence and the dominance of the pole on the phenomenological side) leads to a higher mass. We also predict the masses of the bottom scalar tetraquark resonances with zero and two strange quarks.
\end{abstract}

PACS numbers: $11.55 . \mathrm{Hx}, 12.38 . \mathrm{Lg}, 13.25 .-\mathrm{k}$

Recently the D0 Collaboration reported the observation of a narrow structure, called $X(5568)$, in the decay $X(5568) \rightarrow B_{s}^{0} \pi^{ \pm}[1]$. This is the first observation of a hadronic state with two quarks and two antiquarks of four different flavors and, therefore, can only be explained as a tetraquark or molecular state. The mass and width of the observed state were reported to be: $m=5567.8 \pm 2.9$ (sta) ${ }_{-1.9}^{+0.9}$ (syst) $\mathrm{MeV} / c^{2}$ and $\Gamma=21.9 \pm 6.4(\text { sta })_{-2.5}^{+5.0}$ (syst) $\mathrm{MeV} / c^{2}$. As pointed out in Ref. [1], considering the large mass difference between the mass of the $X(5568)$ and the sum of the $B^{0}$ and $K^{ \pm}$masses, it can be difficult to explain the $X(5568)$ as a molecular state. Therefore, the $X(5568)$ is an excellent candidate for a tetraquark state. If the $B_{s}^{0} \pi^{ \pm}$pair in the $X(5568)$ decay is produced in $S$-wave, its quantum numbers are $J^{P}=0^{+}$as the very narrow $D_{s 0}^{+}(2317)$ state, first discovered in the $D_{s}^{+} \pi^{0}$ decay channel by the BABAR Collaboration [2]. Due to its low mass, the structure of the $D_{s 0}^{* \pm}(2317)$ meson has been extensively debated. It has been interpreted as a $c \bar{s}$ state [3-7], twomeson molecular state [8 [17], $K-D$ - mixing [18], fourquark states [19-22] or a mixture between two-meson and four-quark states [23]. In this paper we use the QCD sum rule (QCDSR) approach [24 28] to investigate the possible four-quark structure for the $X(5568)$ and, therefore, to test if the $X(5568)$ could be the isovector bottom partner of the $D_{s 0}^{+}(2317)$.

The QCDSR for scalar mesons are constructed from the two-point correlation function written in terms of a scalar current $j_{S}$ :

$$
\Pi(q)=i \int d^{4} x e^{i q \cdot x}\left\langle 0\left|T\left[j_{S}(x) j_{S}^{\dagger}(0)\right]\right| 0\right\rangle
$$

The key idea of the QCDSR method is to consider that this correlation function is of dual nature and it depends on the value of the momentum $q$. For large momentum, i.e., short distances, the correlation function can be calculated using perturbative QCD. In this case, the current $j_{S}$ is written in terms of the quark content of the studied mesons. However, since we are interested in studying the properties of hadrons, the relevant energies are lower and contributions from quark condensates, gluon condensates, etc., need to be included in the evaluation of Eq. (1). This can be done by using the Wilson operator product expansion (OPE) of the correlation function. In this case, Eq. (11) is expanded in terms of local condensates and a series of coefficients. The local operators incorporate nonperturbative long-distance effects, while the coefficients, by construction, include only the shortdistance domain and can be determined perturbatively. This way of evaluating the correlation function is customarily named as the calculation on the "OPE side".

At large distances, or, equivalently, small momentum, the currents $j_{S}^{\dagger}$ and $j_{S}$ of Eq. (1) can be interpreted as operators of creation and annihilation of the scalar mesons. In this case, the correlation function is obtained by inserting a complete set of scalar states. This interpretation of the correlation function is called as the "phenomenological side". The assumption made in the QCDSR approach is that there must be a range of $q^{2}$ values in which both descriptions must be equivalent. Calculating the correlation function of Eq. (1) using these two approaches and equating them, it is possible to obtain information about the properties of the hadronic states generated in the system.

In Ref. 22] the $D_{s 0}^{+}(2317)$ state was considered as a diquark-antidiquark tetraquark state and was studied by using the QCDSR approach. A very good agreement with the experimental mass was obtained. Here we follow Ref. [22] to write an analogous but isovector scalar-diquark scalar-antidiquark tetraquark current for $X(5568)$ :

$$
j_{S}=\epsilon_{a b c} \epsilon_{d e c}\left(u_{a}^{T} C \gamma_{5} s_{b}\right)\left(\bar{d}_{d} \gamma_{5} C \bar{b}_{e}^{T}\right),
$$

where $a, b, \quad c, \ldots$ are colour indices, $C$ is the charge conjugation matrix. Of course a scalar-scalar diquark-antidiquark form is not the only possible choice for a scalar tetraquark current, and one could use pseudoscalar-pseudoscalar, vector-vector or axial-axial diquark-antidiquark form. However, it was shown in Ref. [29] that the scalar-scalar type of current gives the more stable results. Therefore, we use the current given by Eq. (2) for the $X^{+}(5568)$. 
The coupling of the state, $X$, to the scalar current, $j_{S}$, can be parametrized in terms of the constant $f_{X}$ as: $\left\langle 0\left|j_{S}\right| X\right\rangle=f_{X}$, therefore, the phenomenological side of Eq. (11) can be written as

$$
\Pi^{\text {phen }}\left(q^{2}\right)=\frac{f_{X}^{2}}{m_{X}^{2}-q^{2}}+\cdots,
$$

where the dots denote the contribution from higher resonances, which is usually parametrized through the introduction of a continuum threshold parameter $s_{0}$ [30].

On the OPE side we work at leading order and consider condensates up to dimension six. We deal with the strange quark as a light one and consider the diagrams up to order $m_{s}$. To keep the bottom quark mass finite, we use the momentum-space expression for the bottom quark propagator. We follow ref. 31 and calculate the light quark part of the correlation function in the coordinate-space, which is then Fourier transformed to the momentum space in $D$ dimensions. The resulting light-quark part is combined with the charm-quark part before it is dimensionally regularized at $D=4$.

We can write the correlation function on the OPE side in terms of a dispersion relation:

$$
\Pi^{O P E}\left(q^{2}\right)=\int_{m_{b}^{2}}^{\infty} d s \frac{\rho(s)}{s-q^{2}},
$$

where the spectral density is given by the imaginary part of the correlation function: $\rho(s)=\frac{1}{\pi} \operatorname{Im}\left[\Pi^{O P E}(s)\right]$. After making a Borel transform on both sides, and transferring the continuum contribution to the OPE side, the sum rule for the scalar meson $X$ can be written as

$$
f_{X}^{2} e^{-m_{X}^{2} / M^{2}}=\int_{m_{b}^{2}}^{s_{0}} d s e^{-s / M^{2}} \rho(s),
$$

where $M$ is the Borel mass and $\rho(s)=\rho^{\text {pert }}(s)+\rho^{m_{s}}(s)+$ $\rho^{\langle\bar{q} q\rangle}(s)+\rho^{\left\langle G^{2}\right\rangle}(s)+\rho^{m i x}(s)+\rho^{\langle\bar{q} q\rangle^{2}}(s)+\rho^{\left\langle G^{3}\right\rangle}(s)$, with

$$
\begin{gathered}
\rho^{\text {pert }}(s)=\frac{1}{2^{10} 3 \pi^{6}} \int_{\Lambda}^{1} d \alpha\left(\frac{1-\alpha}{\alpha}\right)^{3}\left(m_{b}^{2}-s \alpha\right)^{4}, \\
\rho^{m_{s}}(s)=0 \\
\rho^{\langle\bar{q} q\rangle}(s)=\frac{1}{2^{6} \pi^{4}} \int_{\Lambda}^{1} d \alpha \frac{1-\alpha}{\alpha}\left(m_{b}^{2}-s \alpha\right)^{2}[ \\
\left.-\langle\bar{q} q\rangle\left(2 m_{s}+m_{b} \frac{1-\alpha}{\alpha}\right)+m_{s}\langle\bar{s} s\rangle\right] \\
\rho^{\left\langle G^{2}\right\rangle}(s)=\frac{\left\langle g^{2} G^{2}\right\rangle}{2^{10} \pi^{6}} \int_{\Lambda}^{1} d \alpha\left(m_{b}^{2}-s \alpha\right)\left[\frac{m_{b}^{2}}{9}\left(\frac{1-\alpha}{\alpha}\right)^{3}+\right. \\
\left.+\left(m_{b}^{2}-s \alpha\right)\left(\frac{1-\alpha}{2 \alpha}+\frac{(1-\alpha)^{2}}{4 \alpha^{2}}\right)\right],
\end{gathered}
$$

$$
\begin{aligned}
\rho^{\text {mix }}(s)= & \frac{1}{2^{6} \pi^{4}} \int_{\Lambda}^{1} d \alpha\left(m_{b}^{2}-s \alpha\right)\left[-\frac{m_{s}\langle\bar{s} g \sigma \cdot G s\rangle}{6}\right. \\
& +\langle\bar{q} g \sigma \cdot G q\rangle\left(-m_{s}(1-\ln (1-\alpha))\right. \\
& \left.\left.-m_{b} \frac{1-\alpha}{\alpha}\left(1-\frac{1-\alpha}{2 \alpha}\right)\right)\right], \\
\rho^{\langle\bar{q} q\rangle^{2}}(s)= & -\frac{1}{24 \pi^{2}} \int_{\Lambda}^{1} d \alpha\left(2\langle\bar{q} q\rangle\langle\bar{s} s\rangle\left(m_{b}^{2}-s \alpha+m_{b} m_{s}\right)\right. \\
& \left.-\langle\bar{q} q\rangle^{2} m_{b} m_{s}\right), \\
\rho^{\left\langle G^{3}\right\rangle}(s)= & \frac{\left\langle g^{3} G^{3}\right\rangle}{2^{12} 9 \pi^{6}} \int_{\Lambda}^{1} d \alpha\left(\frac{1-\alpha}{\alpha}\right)^{3}\left(3 m_{b}^{2}-s \alpha\right), \quad(12)
\end{aligned}
$$

where the lower limit of the integrations is given by $\Lambda=$ $m_{b}^{2} / s$.

In order to compute the mass of the state, $m_{X}$, we first take the derivative of Eq. (5) with respect to $1 / M^{2}$ and then we divide the result by Eq. (5), obtaining

$$
m_{X}^{2}=\frac{\int_{m_{b}^{2}}^{s_{0}} d s e^{-s / M^{2}} s \rho(s)}{\int_{m_{b}^{2}}^{s_{0}} d s e^{-s / M^{2}} \rho(s)} .
$$

This expression will be used to evaluate the mass of the state.

The numerical values for the quark masses and condensates are listed in Table I] [26, 32 34].

\begin{tabular}{ll}
\hline Parameters & Values \\
\hline$m_{s}$ & $(0.13 \pm 0.03) \mathrm{GeV}$ \\
$m_{b}$ & $(4.24 \pm 0.06) \mathrm{GeV}$ \\
$\langle\bar{q} q\rangle$ & $-(0.23 \pm 0.03)^{3} \mathrm{GeV}^{3}$ \\
$\langle\bar{s} s\rangle$ & $(0.8 \pm 0.2)\langle\bar{q} q\rangle$ \\
$m_{0}^{2}=\langle\bar{q} g \sigma . G q\rangle /\langle\bar{q} q\rangle$ & $0.8 \mathrm{GeV}^{2}$ \\
$\left\langle g^{2} G^{2}\right\rangle$ & $(0.88 \pm 0.25) \mathrm{GeV}^{4}$ \\
$\left\langle g^{3} G^{3}\right\rangle$ & $(0.58 \pm 0.18) \mathrm{GeV}^{6}$ \\
\hline
\end{tabular}

TABLE I. QCD input parameters.

The remaining input to the calculation is the continuum threshold parameter which, in general, is related to the mass of the state to be studied $(X(5568)$, in the present case) as $s_{0} \sim\left(m_{X}+0.5 \mathrm{GeV}\right)^{2}$. Therefore, to start our analysis we choose $\sqrt{s_{0}} \sim 6.0 \mathrm{GeV}$.

In order to determine the values of the Borel mass parameter, we analyze the pole contribution, the OPE convergence and the Borel stability. In the QCDSR approach we extract information only from the ground state, therefore we must ensure that the pole contribution is greater that the continuum contribution. Here we fix the Borel mass in such a way that the pole contribution is always between $80 \%$ and $50 \%$ of the total 


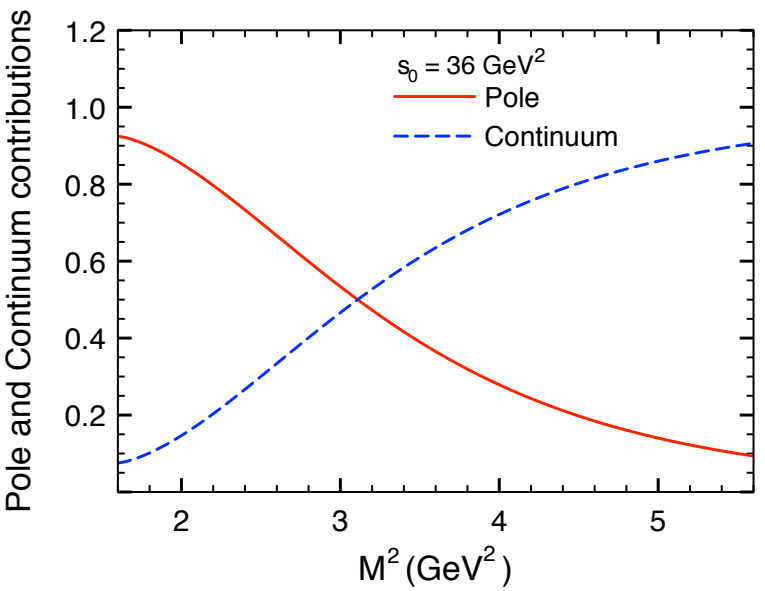

FIG. 1. The pole (solid line) and the continuum (dashed line) contribution for $\sqrt{s_{0}}=6.0 \mathrm{GeV}$.

contribution. From Fig. 1] we can see that this condition is satisfied for values of the Borel mass in the range $2.2 \mathrm{GeV}^{2} \leq M^{2} \leq 3.0 \mathrm{GeV}^{2}$.

In Fig. 2 we plot the ground state mass as a function of $M^{2}$, considering three different values of the threshold parameter. We can see that there is a good $M^{2}$-stability for the Borel window considered. Using the central values of the parameters in Table $\Pi$ and $s_{0}=36 \mathrm{GeV}^{2}$ we get

$$
m_{X} \sim 5.58 \mathrm{GeV}
$$

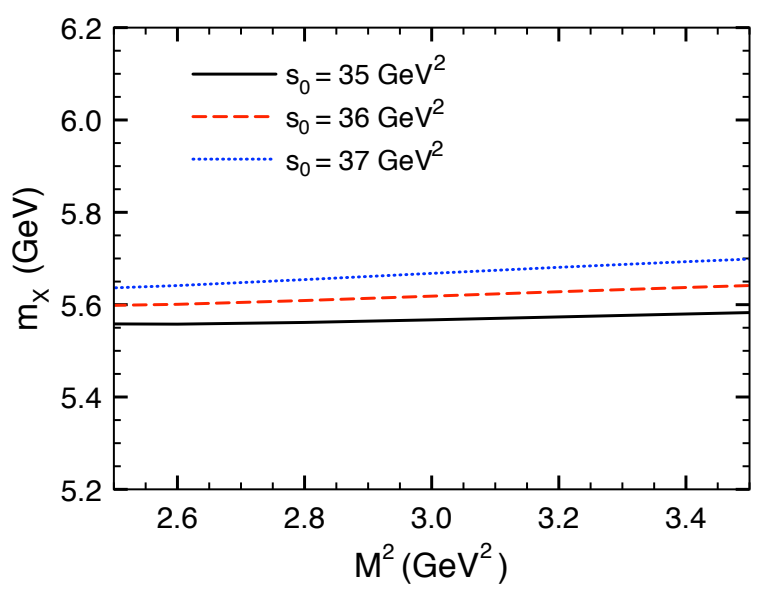

FIG. 2. The $X$ mass as a function of the Borel mass for different values of the continuum threshold: $\sqrt{s_{0}}=5.9 \mathrm{GeV}$ (solid line); $\sqrt{s_{0}}=6.0 \mathrm{GeV}$ (dashed line); $\sqrt{s_{0}}=6.1 \mathrm{GeV}$ (dotted line).

To evaluate the uncertainties inherent to the QCD sum rule approach, we consider the variation of the mass in Borel window, as a function of the continuum threshold, changed within a small range: $5.9 \leq \sqrt{s_{0}} \leq 6.1 \mathrm{GeV}$,

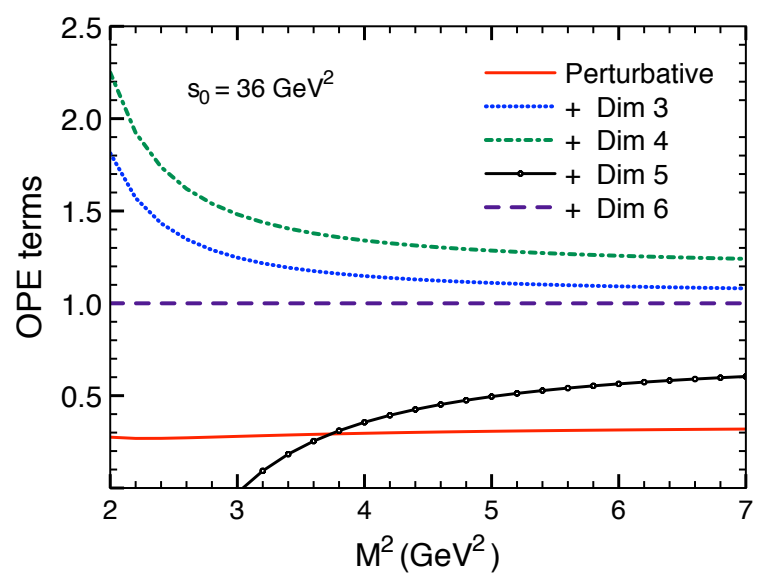

FIG. 3. The OPE convergence in the region $2.0 \leq M^{2} \leq$ $7 \mathrm{GeV}^{2}$ for $s_{0}=36 \mathrm{GeV}$. We start with the relative perturbative contribution (the perturbative contribution divided by the total contribution) and each subsequent line represents the addition of the relative contribution of a condensate of higher dimension in the expansion.

and the quark masses and condensates errors indicated in Table [. Considering these uncertainties we get:

$$
m_{X}=(5.58 \pm 0.17) \mathrm{GeV},
$$

which is in excellent agreement with the experimental mass of the X(5568) determined by the D0 Collaboration [1].

The result in Eq. (15) was obtained considering only the pole dominance and the stability with the Borel mass. There is, however, a stronger constraint to the lower bound of the $M^{2}$, that comes from imposing the OPE convergence. We analyze the convergence of the OPE by comparing the relative contribution of each term given by Eqs. (6) to (12), to the right hand side of Eq. (5). The requirement of a good convergence sets a lower limit to $M^{2}$. This analysis in shown in Fig. 3 .

As can be seen from Fig. 3, there is no OPE convergence in any region allowed by the upper bound given by pole/continuum analysis: $M^{2} \leq 3.0 \mathrm{GeV}^{2}$. This means that the lower bound given by OPE convergence will be higher than the upper bound, and there is no valid "sum rule window" where we can completely trust the results for this current.

To overcome this problem we can consider higher dimension condensates in the OPE side and test if the series starts converging with such contributions. We include the condensates of dimension seven and eight whose expressions are given below:

$$
\begin{aligned}
& \rho^{\langle\bar{s} s\rangle\left\langle G^{2}\right\rangle}(s)=\frac{m_{s}\langle\bar{s} s\rangle\left\langle g^{2} G^{2}\right\rangle}{2^{9} 3^{2} \pi^{4}}\left[3 \int_{\Lambda}^{1} d \alpha+\right. \\
& \left.-\int_{0}^{1} d \alpha \frac{2 m_{b}^{2} \alpha}{(1-\alpha)^{2}} \delta\left(s-\frac{m_{b}^{2}}{1-\alpha}\right)\right]
\end{aligned}
$$




$$
\begin{aligned}
& \rho^{\langle\bar{q} q\rangle\left\langle G^{2}\right\rangle}(s)=-\frac{\langle\bar{q} q\rangle\left\langle g^{2} G^{2}\right\rangle}{2^{8} 3^{2} \pi^{4}}\left[m_{s}+\int_{\Lambda}^{1} d \alpha\left(\left(4 m_{b}+3 m_{s}\right)+\right.\right. \\
& \left.+\frac{3(1-\alpha)}{\alpha}\left(\frac{m_{b}(1-\alpha)}{\alpha}-3 m_{b}\right)\right) \\
& \left.+\int_{0}^{1} d \alpha \frac{m_{b}^{2} \alpha}{(1-\alpha)^{2}}\left(\frac{1}{2}-\frac{m_{b} \alpha}{1-\alpha}\right) \delta\left(s-\frac{m_{b}^{2}}{1-\alpha}\right)\right] \\
& \rho^{\langle\bar{q} q\rangle\langle\bar{q} G q\rangle}(s)=\frac{m_{b} m_{s}\langle\bar{q} q\rangle\langle\bar{q} g \sigma \cdot G q\rangle}{2^{4} 3 \pi^{2}}\left[\int_{0}^{1} d \alpha \frac{1}{1-\alpha} \times\right. \\
& \left.\times \delta\left(s-\frac{m_{b}^{2}}{1-\alpha}\right)-2 \delta\left(s-m_{b}^{2}\right)\right], \\
& \rho^{\langle\bar{s} s\rangle\langle\bar{q} G q\rangle}(s)=-\frac{\langle\bar{s} s\rangle\langle\bar{q} g \sigma \cdot G q\rangle}{2^{5} 3 \pi^{2}}\left[2-m_{b} m_{s} \delta\left(s-m_{b}^{2}\right)+\right. \\
& \left.+\int_{0}^{1} d \alpha \frac{m_{b} m_{s}}{1-\alpha} \delta\left(s-\frac{m_{b}^{2}}{1-\alpha}\right)\right], \\
& \rho^{\langle\bar{q} q\rangle\langle\bar{s} G s\rangle}(s)=-\frac{\langle\bar{q} q\rangle\langle\bar{s} g \sigma \cdot G s\rangle}{2^{5} 3^{2} \pi^{2}}\left(6+\delta\left(s-m_{b}^{2}\right)\right), \\
& \rho^{\left\langle g^{4} G^{4}\right\rangle}(s)=-\frac{\left\langle g^{4} G^{4}\right\rangle}{2^{14} 3 \pi^{6}}\left[\int_{\Lambda}^{1} d \alpha+\frac{2}{3} m_{b}^{2} \int_{0}^{1} d \alpha \frac{\alpha}{(1-\alpha)^{2}} \times\right. \\
& \left.\times \delta\left(s-\frac{m_{b}^{2}}{1-\alpha}\right)\right] \text {. }
\end{aligned}
$$

On continuing with our analysis we find that, even after considering condensates up to dimension eight, a valid "sum rule window" exists only for values of $s_{0} \geq 46 \mathrm{GeV}^{2}$. In Figs. 4 and 5 we show the OPE convergence and the pole versus continuum contribution for $s_{0} \geq 46 \mathrm{GeV}^{2}$.

From Fig. 4 we can see that there is an OPE convergence, the dimension eight condensate contribution is smaller than $20 \%$ of the total contribution, only for values of $M^{2} \geq 4.4 \mathrm{GeV}^{2}$. On the other hand, from Fig. [5 we can see that the pole contribution is bigger than the continuum contribution for values of $M^{2} \leq 4.5 \mathrm{GeV}^{2}$. Although very small, there exists a valid Borel window in the region $4.4 \mathrm{GeV}^{2} \leq M^{2} \leq 4.5 \mathrm{GeV}^{2}$, which provides a good "sum rule" to extract a reliable value for the mass of the state.

In Fig. 6, we show the resulting value for the mass of the state, as a function of the Borel mass, for three different values of the continuum threshold. The crosses in the figure indicate the "sum rule window".

Finally, considering all the restrictions described above we get:

$$
m_{X}=(6.39 \pm 0.10) \mathrm{GeV},
$$

which is not in agreement with the experimental mass of the $X(5568)$ determined by the D0 Collaboration [1].

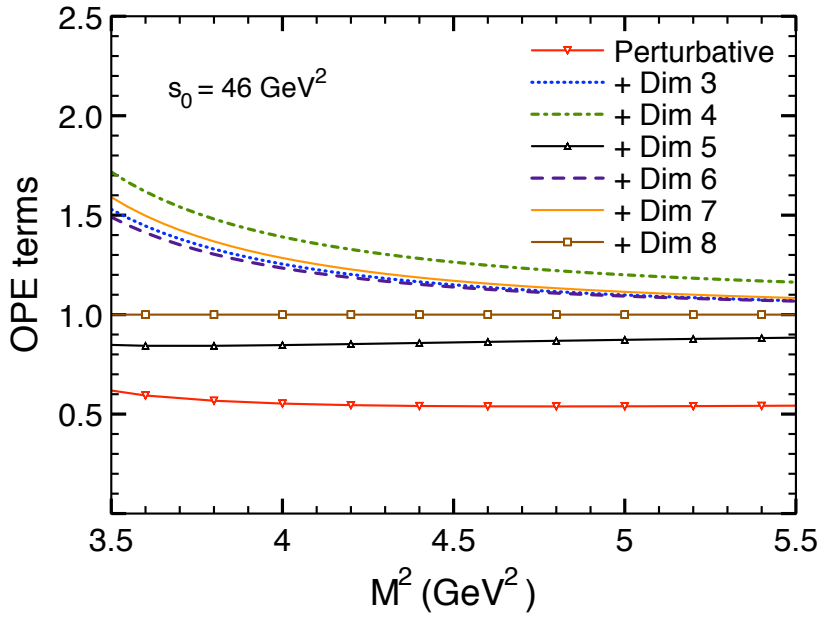

FIG. 4. The OPE convergence in the region $3.5 \leq M^{2} \leq$ $5.5 \mathrm{GeV}^{2}$ for $s_{0}=46 \mathrm{GeV}$. We start with the relative perturbative contribution (the perturbative contribution divided by the total contribution) and each subsequent line represents the addition of the relative contribution of a condensate of higher dimension in the expansion.

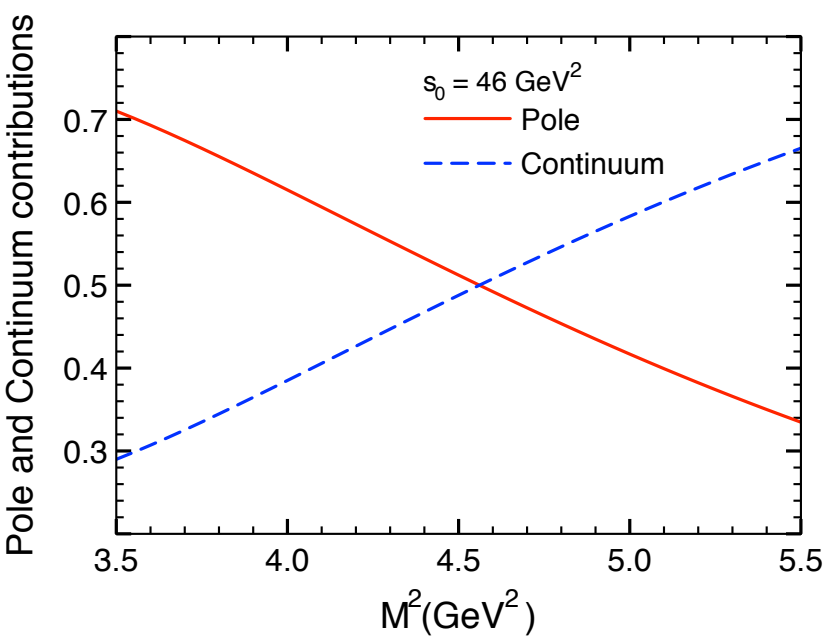

FIG. 5. The pole (solid line) and the continuum (dashed line) contribution for $s_{0}=46.0 \mathrm{GeV}^{2}$.

As a matter of fact, recently the $\mathrm{LHCb}$ Collaboration has not confirmed the observation of the $X(5568)$. In their preliminary analysis [35] no structure is found in the $B_{s}^{0} \pi^{ \pm}$mass spectrum from the $B_{s}^{0} \pi^{+}$threshold up to $M_{B_{s}^{0} \pi^{+}} \leq 5700 \mathrm{GeV}$. More analyses are required to clarify this situation. Our work predicts a tetraquark state decaying in this channel with a mass around 6.39 $\mathrm{GeV}$.

The uncertainty given in Eq. (22) is only related with the range of values of the Borel mass window, a small variation in the continuum threshold, $46 \leq s_{0} \leq 50 \mathrm{GeV}^{2}$, 


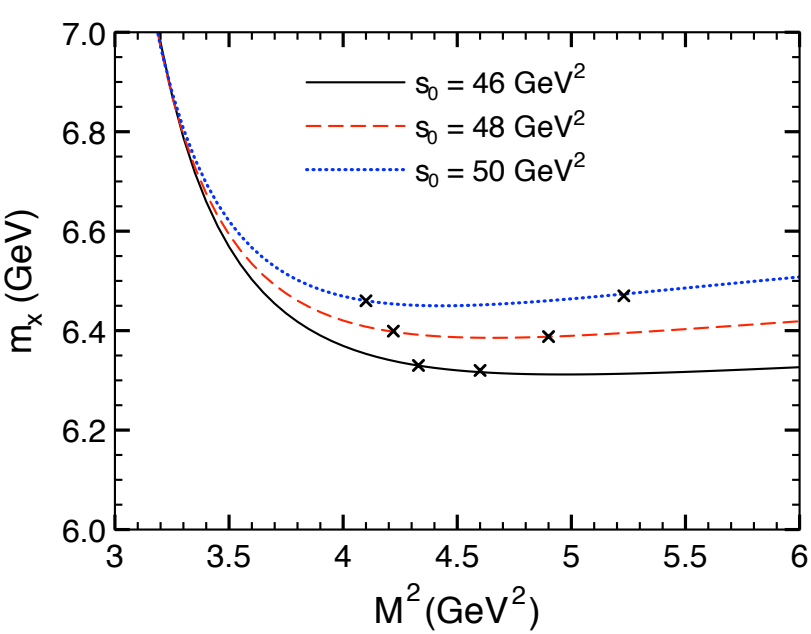

FIG. 6. The $X$ mass as a function of the Borel mass for different values of the continuum threshold: $s_{0}=46 \mathrm{GeV}^{2}$ (solid line); $s_{0}=48 \mathrm{GeV}^{2}$ (dashed line); $s_{0}=50 \mathrm{GeV}^{2}$ (dotted line). The crosses in the figure indicate the allowed Borel window.

and the quark masses and condensates errors indicated in Table प The difference between the values in Eqs. (15) and (22) can be associated mainly with the change in the value of the continuum threshold. However, as discussed above, there is no allowed Borel window for values of $s_{0} \leq 46 \mathrm{GeV}^{2}$. Therefore, the result obtained with $s_{0} \sim 36 \mathrm{GeV}^{2}$ given in Eq. (15), although being obtained in a Borel region where one has pole dominance, can not be trusted. This result illustrates very well how we can reproduce the mass of a given state and then after a more careful analysis conclude that the state is not the particle associated with the chosen current. We also would like to point out that the difference between $\sqrt{s_{0}}=\sqrt{48} \mathrm{GeV}$ and the result in Eq. (22) is about $0.5 \mathrm{GeV}$, as the general supposition of the QCDSR approach for the start of the continuum threshold. It is also important to notice that the difference: $\sqrt{s_{0}}-m_{X}$ increases with the value of the continuum threshold. As an example, for $s_{0} \sim 64 \mathrm{GeV}^{2}$ we get $m_{X} \sim 6.7 \mathrm{GeV}$ which implies $\sqrt{s_{0}}-m_{X} \sim 1.3 \mathrm{GeV}$, much larger than $0.5 \mathrm{GeV}$. This could be an indication that there is a contribution from higher resonances below the continuum threshold and, therefore, once again, the estimated mass can not be trusted. Therefore, to fix a "good range" of the values of $s_{0}$ we test if it provides an allowed Borel window (where both constraints of the pole dominance and the ope convergence are satisfied), and that the value of the obtained mass falls within the range $0.4 \mathrm{GeV}$ to $0.6 \mathrm{GeV}$ smaller than $\sqrt{s_{0}}$. Using these criteria, we have obtained $s_{0}$ in the range $46 \leq s_{0} \leq 50 \mathrm{GeV}^{2}$.

We can extend the formalism to study bottom scalar mesons states that contain zero and two strange quarks. In order to calculate the correlation function for these

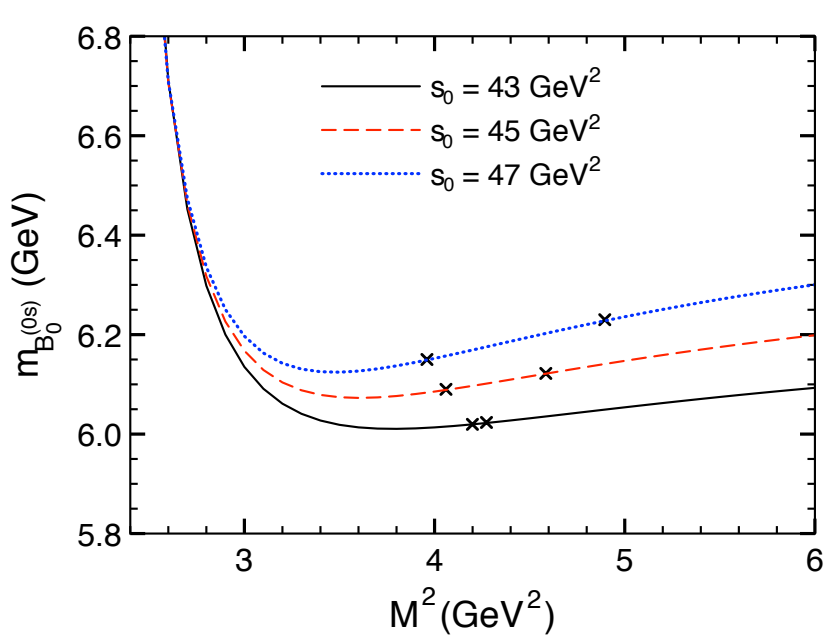

FIG. 7. The $B_{0}^{0 s}$ mass as a function of the Borel mass for different values of the continuum threshold: $s_{0}=43 \mathrm{GeV}^{2}$ (solid line); $s_{0}=45 \mathrm{GeV}^{2}$ (dashed line); $s_{0}=47 \mathrm{GeV}^{2}$ (dotted line). The crosses in the figure indicate the allowed Borel window.

states we use the following interpolating fields for these states (zero and two strange quarks, respectively):

$$
\begin{aligned}
j_{0} & =\epsilon_{a b c} \epsilon_{d e c}\left(u_{a}^{T} C \gamma_{5} q_{b}\right)\left(\bar{d}_{d} \gamma_{5} C \bar{b}_{e}^{T}\right), \\
j_{s s} & =\epsilon_{a b c} \epsilon_{d e c}\left(u_{a}^{T} C \gamma_{5} s_{b}\right)\left(\bar{s}_{d} \gamma_{5} C \bar{b}_{e}^{T}\right),
\end{aligned}
$$

where $q$ represents the quark $u$ or $d$ according to the charge of the meson. The expression for the resulting spectral densities are given in Appendix A.

We call $B_{0}^{(0 s)}$ and $B_{0}^{(2 s)}$ the scalar bottom tetraquark mesons represented by $j_{0}$ and $j_{s s}$ respectively (Eq. 231). In Figs. 7 and 8 we show the masses of the states as a function of the Borel mass for different values of the continuum threshold, and as the previous case there is good $M^{2}$-stability in the allowed Borel window, represented by the crosses in these figures.

The values obtained for the masses of the states are:

$$
\begin{gathered}
m_{B_{0}^{0 s}}=6.10 \pm 0.16 \mathrm{GeV}, \\
m_{B_{0}^{2 s}}=6.39 \pm 0.17 \mathrm{GeV} .
\end{gathered}
$$

The sources of errors are the same that were used for the strange-bottom scalar meson.

Comparing the results of the masses in Eqs. (22) and (25) we can see that the $X$ (the state with one strange quark) and $B_{0}^{(2 s)}$ resonance masses are basically degenerated, while the mass of $B_{0}^{(0 s)}$ is around $300 \mathrm{MeV}$ smaller than the others. The same behavior is observed for the scalar mesons in the charm sector, as it was pointed out in Ref. 22]. The increase in the mass is expected with the inclusion of one strange quark (from zero to one strange quarks). The fact this is not observed when one goes from one to two strange quarks can be traced when we 


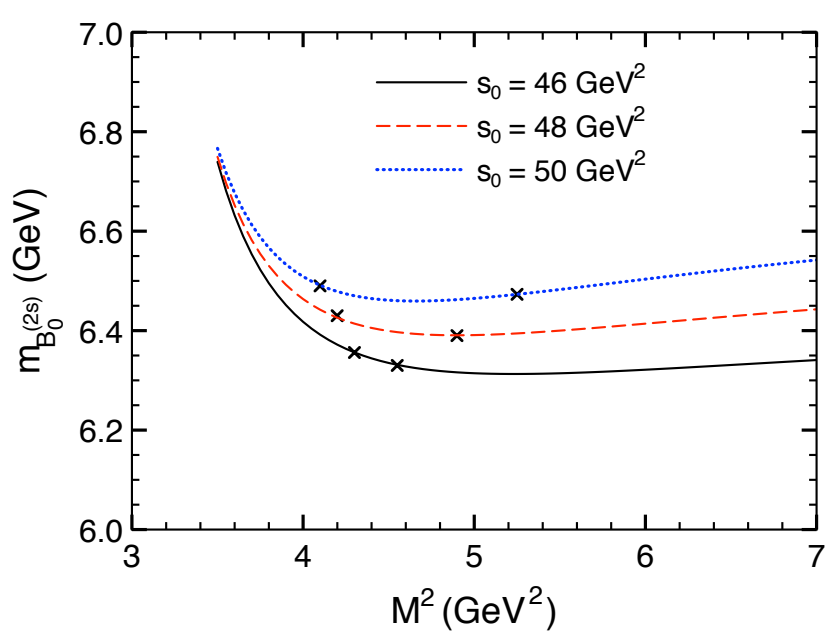

FIG. 8. The $B_{0}^{2 s}$ mass as a function of the Borel mass for different values of the continuum threshold: $\sqrt{s_{0}}=46 \mathrm{GeV}^{2}$ (solid line); $s_{0}=48 \mathrm{GeV}^{2}$ (dashe line); $s_{0}=50 \mathrm{GeV}^{2}$ (dotted line). The crosses in the figure indicate the allowed Borel window.

compare the contribution of the quark condensate term, that is smaller for the $B_{0}^{(2 s)}$, but this is compensated by the inclusion of a term $\rho^{m_{s}}$ (Eq. A8).

In summary, we have presented a QCD sum rule study for a bottom scalar meson considered as diquarkantidiquark state. The motivation of the study is to look for a possible state associated with the recently claimed $X(5568)$ by the D0 Collaboration. We find that it is possible to obtain a stable mass in agreement with the state found by the D0 collaboration while satisfying the condition of the pole dominance on the phenomenological side, but by sacrificing the simultaneous constraint of the OPE convergence. This last missing ingredient casts a doubt on the reliability of the result, leading us to conclude that the $X(5568)$ state can not be represented by the scalar tetraquark current. We find that a rigorous application of QCD sum rule constraints leads to a higher mass. Thus, we predict the existence of a scalar bottomstrange tetraquark state with a mass around $6.4 \mathrm{GeV}$. We also have obtained the masses for the resonances with zero and two strange quarks, which we call $B_{0}^{0 s}$ and $B_{0}^{2 s}$. These resonances have also not been observed yet, but our calculations show that they should exist.

Acknowledgements: This work has been supported by $\mathrm{CNPq}$ and FAPESP.
Appendix A: The spectral densities for resonances with zero and two strange quarks

The expressions (6), (9) and (12) are common to the three resonances. Next, we list the other terms that are not common.

From $j_{0}$ we get:

$$
\rho^{m_{s}}(s)=0
$$

$$
\begin{gathered}
\rho^{\langle\bar{q} q\rangle}(s)=-\frac{m_{b}\langle\bar{q} q\rangle}{2^{6} \pi^{4}} \int_{\Lambda}^{1} d \alpha\left(\frac{1-\alpha}{\alpha}\right)^{2}\left(m_{b}^{2}-s \alpha\right)^{2}, \text { (A2) } \\
\rho^{m i x}(s)=\frac{m_{b}\langle\bar{q} g \sigma \cdot G q\rangle}{2^{6} \pi^{4}}\left[\frac{1}{2} \int_{\Lambda}^{1} d \alpha\left(\frac{1-\alpha}{\alpha}\right)^{2}\left(m_{b}^{2}-s \alpha\right)+\right. \\
\left.-\int_{\Lambda}^{1} d \alpha \frac{1-\alpha}{\alpha}\left(m_{b}^{2}-s \alpha\right)\right], \\
\rho^{\langle\bar{q} q\rangle^{2}}(s)=-\frac{\langle\bar{q} q\rangle^{2}}{12 \pi^{2}} \int_{\Lambda}^{1} d \alpha\left(m_{b}^{2}-s \alpha\right) . \\
\rho^{\langle\bar{s} s\rangle\left\langle G^{2}\right\rangle}(s)=\frac{\langle\bar{s} s\rangle\left\langle g^{2} G^{2}\right\rangle}{2^{8} 3^{2} \pi^{4}}\left[-\int_{0}^{1} d \alpha \frac{m_{b}^{3} \alpha^{2}}{(1-\alpha)^{3}} \times\right. \\
\left.+\int_{\Lambda}^{1} d \alpha\left(\frac{(1-\alpha)(12 \alpha-3)}{\alpha^{2}} \times m_{b}-\frac{8 m_{b}}{2}\right)\right], \\
\rho^{\left\langle G^{4}\right\rangle}(s)=\frac{\left\langle g^{2}\right\rangle}{2^{13} 3^{2} \pi^{6}}(s)=0, \\
\times \delta\left(s-\frac{3}{2} \int_{\Lambda}^{1} d \alpha-\int_{0}^{1} d \alpha \frac{m_{b}^{2}}{(1-\alpha}\right)
\end{gathered}
$$

From $j_{s s}$ we get:

$$
\begin{gathered}
\rho^{m_{s}}(s)=-\frac{m_{s} m_{b}}{2^{8} 3 \pi^{6}} \int_{\Lambda}^{1} d \alpha\left(\frac{1-\alpha}{\alpha}\right)^{3}\left(m_{b}^{2}-s \alpha\right)^{3}, \\
\left.\rho^{\langle\bar{q} q\rangle}(s)=\frac{1}{2^{6} \pi^{4}} \int_{\Lambda}^{1} d \alpha \frac{1-\alpha}{\alpha}\left(m_{b}^{2}-s \alpha\right)^{2}\right] \\
\left.\langle\bar{s} s\rangle\left(2 m_{s}-m_{b} \frac{1-\alpha}{\alpha}\right)-2 m_{s}\langle\bar{q} q\rangle\right], \\
\rho^{m i x}(s)=\frac{1}{2^{6} \pi^{4}} \int_{\Lambda}^{1} d \alpha\left(m_{b}^{2}-s \alpha\right)\left[\frac { \langle \overline { s } g \sigma \cdot G s \rangle } { 2 } \left(\frac{m_{s}}{3}\right.\right. \\
\left.-m_{s} \frac{1-\alpha}{\alpha}-m_{b} \frac{1-\alpha}{\alpha}\left(1-\frac{1-\alpha}{2 \alpha}\right)\right) \\
\left.-m_{s}\langle\bar{q} g \sigma \cdot G q\rangle(1-\ln (1-\alpha))\right]
\end{gathered}
$$




$$
\begin{aligned}
& \rho^{\langle\bar{q} q\rangle^{2}}(s)=\frac{\langle\bar{s} s\rangle}{48 \pi^{2}}\left[\int _ { \Lambda } ^ { 1 } d \alpha \left(4\langle\bar{q} q\rangle\left(s \alpha-m_{b}^{2}\right)+2 m_{s} m_{b} \times\right.\right. \\
& \left.\times(2\langle\bar{q} q\rangle-\langle\bar{s} s\rangle))+m s^{2}(\langle\bar{s} s\rangle-2\langle\bar{q} q\rangle)\right], \\
& \rho^{\langle\bar{q} q\rangle\left\langle G^{2}\right\rangle}(s)=\frac{m_{s}\langle\bar{q} q\rangle\left\langle g^{2} G^{2}\right\rangle}{2^{8} 3^{2} \pi^{4}}\left[\int_{0}^{1} d \alpha \frac{2 m_{b}^{2} \alpha}{(1-\alpha)^{2}}(1+\right. \\
& \left.+\frac{m_{b} m_{s}}{(1-\alpha) M^{2}}\right) \delta\left(s-\frac{m_{b}^{2}}{(1-\alpha)}\right)-3 \int_{\Lambda}^{1} d \alpha+ \\
& \left.-\left(1+m_{b} m_{s} \delta\left(s-m_{b}^{2}\right)\right)\right] \\
& \rho^{\langle\bar{s} s\rangle\left\langle G^{2}\right\rangle}(s)=\frac{\langle\bar{s} s\rangle\left\langle g^{2} G^{2}\right\rangle}{2^{8} 3^{2} \pi^{4}}\left[\frac{3 m_{s}}{2}-\int_{0}^{1} d \alpha \frac{m_{b}^{2} \alpha}{(1-\alpha)^{2}} \times\right. \\
& \times\left(\frac{\left(\alpha M^{2}+m_{s}^{2}\right) m_{b}}{(1-\alpha) M^{2}}+2 m_{s}\right) \delta\left(s-\frac{m_{b}^{2}}{1-\alpha}\right) \\
& \left.+\int_{\Lambda}^{1} d \alpha\left(\frac{3(1-\alpha)(4 \alpha-1) m_{b}}{\alpha^{2}}+\frac{3 m_{s}-8 m_{b}}{2}\right)\right], \\
& \rho^{\left\langle G^{4}\right\rangle}(s)=\frac{\left\langle g^{4} G^{4}\right\rangle}{2^{13} 3^{2} \pi^{6}}\left[\frac{3}{2} \int_{\Lambda}^{1} d \alpha-\int_{0}^{1} d \alpha \frac{m_{b}^{2} \alpha}{(1-\alpha)^{2}}(1+\right. \\
& \left.\left.+\frac{m_{b} m_{s}}{(1-\alpha) M^{2}}\right) \delta\left(s-\frac{m_{b}^{2}}{1-\alpha}\right)\right] \text {. } \\
& \rho^{\langle\bar{s} s\rangle\langle\bar{s} g \sigma \cdot G s\rangle}(s)=m_{b} m_{s} \frac{\langle\bar{s} s\rangle\langle\bar{s} g \sigma \cdot G s\rangle}{2^{5} 3^{2} \pi^{2}}\left[\frac{2 M^{2}-m_{b} m_{s}}{M^{2}}\right. \\
& \left.\times \delta\left(s-m_{b}^{2}\right)-\int_{0}^{1} d \alpha \frac{3}{1-\alpha} \delta\left(s-\frac{m_{b}^{2}}{1-\alpha}\right)\right], \\
& \left.\left.\times \delta\left(s-\frac{m_{b}^{2}}{1-\alpha}\right)+\left(m_{b} m_{s}-3\right) \delta\left(s-m_{b}^{2}\right)\right)-\frac{3}{2}\right],
\end{aligned}
$$

[1] V. M. Abazov et al. [D0 Collaboration], arXiv:1602.07588 [hep-ex].

[2] BABAR Coll., B. Auber et al., Phys. Rev. Lett. 90, 242001 (2003); Phys. Rev. D69, 031101 (2004).

[3] Y.-B. Dai, C.-S. Huang, C. Liu and S.-L. Zhu, Phys. Rev. D68, 114011 (2003).

[4] G.S. Bali, Phys. Rev. D68, 071501(R) (2003).

[5] A. Dougall, R.D. Kenway, C.M. Maynard and C. McNeile, Phys. Lett. B569, 41 (2003).

[6] A. Hayashigaki and K. Terasaki, hep-ph/0411285.

[7] S. Narison, Phys. Lett. B605, 319 (2005).

[8] T. Barnes, F.E. Close and H.J. Lipkin, Phys. Rev. D68, 054006 (2003).

[9] A.P. Szczepaniak, Phys. Lett. B567, 23 (2003).

[10] E. E. Kolomeitsev and M. F. M. Lutz, Phys. Lett. B 582, 39 (2004).

[11] F. K. Guo, P. N. Shen, H. C. Chiang, R. G. Ping and B. S. Zou, Phys. Lett. B 641, 278 (2006).

[12] D. Gamermann, E. Oset, D. Strottman and M. J. Vicente Vacas, Phys. Rev. D 76, 074016 (2007).

[13] F. K. Guo, C. Hanhart and U. G. Meissner, Eur. Phys. J. A 40, 171 (2009).

[14] M. Cleven, F. K. Guo, C. Hanhart and U. G. Meissner, Eur. Phys. J. A 47, 19 (2011).

[15] M. Cleven, H. W. Griesshammer, F. K. Guo, C. Hanhart and U. G. Meissner, Eur. Phys. J. A 50, 149 (2014).

[16] A. Faessler, T. Gutsche, S. Kovalenko and V. E. Lyubovitskij, Phys. Rev. D 76, 014003 (2007).

[17] A. Faessler, T. Gutsche, V. E. Lyubovitskij and Y. L. Ma,
Phys. Rev. D 76, 014005 (2007).

[18] E. van Beveren and G. Rupp, Phys. Rev. Lett. 91, 012003 (2003).

[19] H.-Y. Cheng and W.-S. Hou, Phys. Lett. B566, 193 (2003).

[20] K. Terasaki, Phys. Rev. D68, 011501(R) (2003).

[21] L. Maiani, F. Piccinini, A.D. Polosa, V. Riquer, Phys. Rev. D71, 014028 (2005).

[22] M. E. Bracco, A. Lozea, R. D. Matheus, F. S. Navarra and M. Nielsen, Phys. Lett. B 624, 217 (2005).

[23] T. Browder, S. Pakvasa and A.A. Petrov, Phys. Lett. B578, 365 (2004).

[24] M.A. Shifman, A.I. and Vainshtein and V.I. Zakharov, Nucl. Phys. B 147, 385 (1979).

[25] L.J. Reinders, H. Rubinstein and S. Yazaki, Phys. Rept. 127, 1 (1985).

[26] For a review and references to original works, see e.g., S.Narison, $Q C D$ as a theory of hadrons, Cambridge Monogr. Part. Phys. Nucl. Phys. Cosmol. 17, 1 (2002) hep-h/0205006; QCD spectral sum rules, World Sci. Lect. Notes Phys. 26, 1 (1989); Acta Phys. Pol. B26, 687 (1995); Riv. Nuov. Cim. 10N2, 1 (1987); Phys. Rept. 84, 263 (1982).

[27] M. Nielsen, F. S. Navarra and S. H. Lee, Phys. Rept. 497, 41 (2010).

[28] M. Nielsen and F. S. Navarra, Mod. Phys. Lett. A 29, 1430005 (2014).

[29] H. Kim and Y. Oh, Phys. Rev. D 72, 074012 (2005).

[30] B. L. Ioffe, Nucl. Phys. B188, 317 (1981); B191, 591(E) 
(1981).

[31] H. Kim, S.H. Lee and Y. Oh, Phys. Lett. B595, 293 (2004).

[32] S. Narison, Nucl. Phys. Proc. Suppl. 86 (2000) 242 (hepph / 9911454); S. Narison, hep-ph / 0202200; S. Narison, hep-ph / 0510108; S. Narison, Phys. lett. B341 (1994) 73; H.G. Dosch and S. Narison, Phys. lett. B417 (1998) 173; S. Narison, Phys. lett. B216 (1989) 191.
[33] S. Narison, Phys. Lett. B466 , 345 (1999).

[34] S. Narison, Phys. Lett. B361 , 121 (1995); S. Narison, Phys. Lett. B387, 162 (1996). S. Narison, Phys.Lett. B624 (2005) 223.

[35] The LHCb Collaboration, [LHCb Collaboration]:"Search for structure in the $B_{s}^{0} \pi^{ \pm}$invariant mass spectrum", LHCb-CONF-2016-004, CERN-LHCb-CONF-2016-004. 


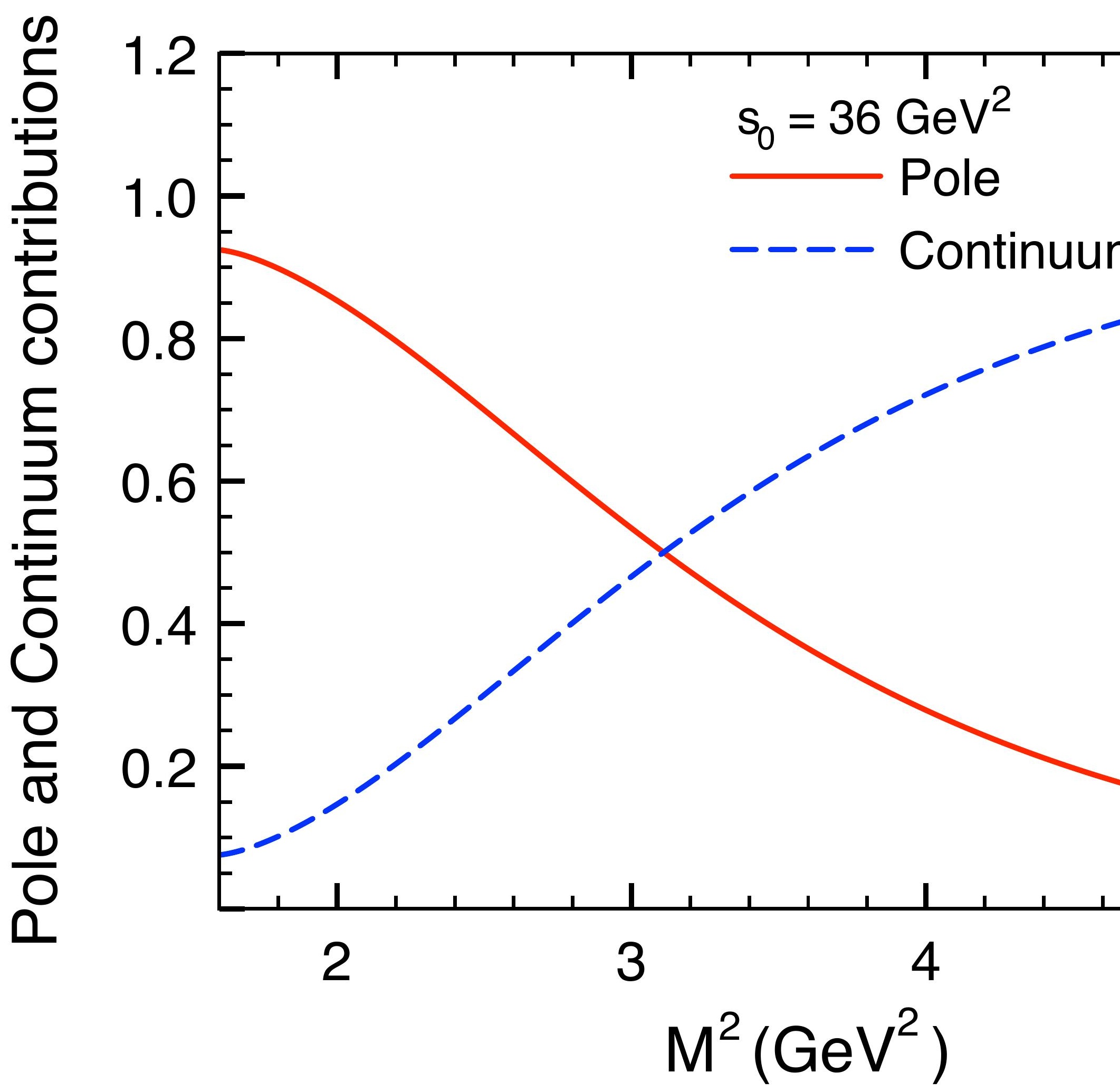

